\title{
Temas de interés para los asistentes a la expo tutoría: nuevas áreas de oportunidad
}

\section{Topics of interest for expo tutoring attendees: new areas of opportunity}

\author{
LUCIO, Rodolfo†' \& SESENTO, Leticia*" \\ 'Facultad de Medicina Veterinaria y Zootecnia de la Universidad Michoacana de San Nicolás de Hidalgo. Calle de Santiago \\ Tapia 403, Centro, 58000 Morelia, Mich. \\ "Colegio Primitivo y Nacional de San Nicolás de Hidalgo. Av Francisco I. Madero Pte 351, Centro histórico de Morelia, \\ 58000 Morelia, Mich.
}

ID $1^{\text {er }}$ Autor: Rodolfo, Lucio / ORC ID: 0000-0002-0535-3652, Researcher ID Thomson: X-2391-2018

ID $1^{\text {er }}$ Coautor: Leticia, Sesento / ORC ID: ORC ID: 0000-0002-6456058X, Researcher ID Thomson: S-6997-2018, CVU CONACYT ID: 449302

\begin{abstract}
Resumen
El presente trabajo muestra los resultados de un estudio realizado en el desarrollo de la Tercera Expo Tutoría. El objetivo fue identificar los temas de interés para los estudiantes en las actividades de tutoría. La investigación se desarrolló bajo un enfoque cuantitativo, descriptivo. Se encuestó a 105 asistentes en muestreo aleatorio simple. Se utilizó una encuesta en la que se pidió la impresión del evento y se les solicitó escribir el o los temas que les gustaría que se les impartiera en el próximo evento y para rescátalos en sus sesiones de tutoría. Los resultados muestran que los temas de mayor interés son violencia (familiar, escolar y en el noviazgo) $19 \%$ y primeros auxilios médicos con el mismo porcentaje (19\%), los temas de sexualidad y estrés con $16 \%$ respectivamente, seguido de orientación vocacional y drogas con un $9 \%$ cada uno, la relación maestro alumno y autoestima con un $6 \%$ respectivamente. La re significación de las practicas docentes, actividad tutorial, el clima institucional y el trabajo colaborativo son los aspectos a trabajar con los jóvenes.
\end{abstract}

\begin{abstract}
The present work shows the results of a study carried out in the development of the Third Expo Tutoría. The objective was to identify topics of interest to students in tutoring activities. The research was developed under a quantitative, descriptive approach. We surveyed 105 attendees in simple random sampling. A survey was used in which the impression of the event was requested and they were asked to write the topic (s) they would like to be taught in the next event and to rescue them in their tutorial sessions. The results show that the topics of greatest interest are violence (family, school and dating) 19\% and medical first aid with the same percentage (19\%), sexuality and stress issues with $16 \%$ respectively, followed by vocational guidance and drugs with $9 \%$ each, the student teacher and self-esteem ratio with $6 \%$ respectively. The resignification of teaching practices, tutorial activity, institutional climate and collaborative work are the aspects to work with young people.
\end{abstract}

\footnotetext{
* Correspondencia del Autor (Correo electrónico: leticiasesentogarcia@yahoo.com.mx)

$\dagger$ Investigador contribuyendo como primer autor.
} 


\section{Introducción}

La tutoría tiene dos objetivos generales (Jara, 2010):

- Coadyuvar al desarrollo las actitudes de los estudiantes que los hace ser mejores seres humanos.

- Favorecer el desempeño académico de los alumnos a través de acciones personalizadas o grupales.

- Contribuir a la formación integral de los estudiantes.

Así, por sus objetivos, puede establecerse que las acciones que se realicen deben estar encaminadas a que los estudiantes consoliden sus conocimientos y habilidades, pero también desarrollen elementos actitudinales y valores que consoliden su formación humana. Es por ello la capacitación continua y la evaluación de los programas de tutoría son indispensables para una mejora y para brindar apoyo en las diversas áreas que el alumno requiera, con ello se contribuye a la formación integral y al desarrollo personal de los jóvenes estudiantes.

\section{Justificación}

La Educación Media Superior busca fortalecer el desarrollo académico y personal de los jóvenes para brindarle las herramientas necesarias para el ingreso a la Educación Superior. Para ello, la Tutoría busca promover una serie de acciones encaminadas a fortalecer el desarrollo integral de los estudiantes, mediante un seguimiento personalizado desde que ingresan al Bachillerato hasta que concluyan este nivel educativo, para apoyarles en la resolución o canalización de sus problemas académicos, pedagógicos $\mathrm{o}$ personales que surjan durante su proceso de formación en este nivel educativo para que con ello se disminuya el ausentismo, deserción, reprobación, violencia escolar, entre otros problemas propios del contexto.

\section{Problema}

Actualmente las Instituciones de Educación Media Superior se enfrentan a diversos desafíos no sólo académicos, sino sociales, personales y familiares de los jóvenes, lo cual, impacta su desempeño y desarrollo académico.
Es por ello, que basándose en sus necesidades las Instituciones y en específico la Coordinación de Tutoría debe capacitar a sus tutores en las diversas temáticas que surgen en su entorno, es por ello, la importancia de medidas como la que aquí se muestra para brindar apoyo al docente que ya detectó el área de oportunidad con su tutorado y busca la capacitación para poder atenderlo.

\section{Objetivo}

Conocer los temas de interés para los docentes o tutores para los cuales buscan ser capacitados y poder implementarlos en su trabajo docente y tutorial con los jóvenes de educación media superior.

\section{Marco teórico}

\section{Inicios}

A partir del Ciclo Escolar 2009-2010 la Dirección General del Bachillerato incorporó en su plan de estudios los principios básicos de la Reforma Integral de la Educación Media Superior cuyo propósito es fortalecer y consolidar la identidad de este nivel educativo, en todas sus modalidades y subsistemas; proporcionar una educación pertinente y relevante al estudiante que le permita establecer una relación entre la escuela y su entorno; y facilitar el tránsito académico de los estudiantes entre los subsistemas y las escuelas.

Basándose en lo anterior surge la Tutoría, la cual, tiene como propósito apoyar en la prevención de los problemas de rezago y deserción, buscando subir los índices de eficiencia terminal, mediante acciones encaminadas a mejorar el clima de convivencia en los planteles y abordando los problemas académicos de las alumnas y alumnos. Esto implica favorecer la superación académica, promover los procesos de pensamiento, la toma de decisiones, la solución de problemas, el sentido de la responsabilidad en el alumnado, así como fortalecer el desarrollo de competencias a través de la integración, la retroalimentación del proceso educativo, la motivación del alumnado y el apoyo académico, por medio de la mediación de la persona responsable de la Acción Tutorial al contribuir en la adquisición de aprendizajes para construir un proyecto de vida. (DGB, 2012) 
La tutoría para estudiantes de este nivel académico, pretende significar un acompañamiento personalizado, es decir, esta acción refiere la atención que un profesor capacitado como tutor realiza sobre el estudiante, con el propósito de que éste alcance su pleno desarrollo, en cuanto a su crecimiento y madurez, y a la manifestación de actitudes de responsabilidad y de libertad. Desde sus orígenes, la acción tutorial deja ver una propensión hacia la atención individualizada a cargo de alguien que-demostradamente- cuenta con una mayor capacidad para apoyar el aprendizaje del estudiante (Romo, 2011)

\section{Concepto}

La Asociación Nacional de Universidades e Instituciones de Educación Superior (2001) propone una definición de tutoría señalada como: "un proceso de acompañamiento durante la formación de los estudiantes, que se concreta mediante la atención personalizada a un alumno o a un grupo reducido de alumnos, por su parte de académicos competentes y formados para esta función, apoyándose conceptualmente en las teorías del aprendizaje más que en las de la enseñanza" (p. 23).

En México, esta definición tuvo un gran impacto y se puede considerar como la definición "oficial" de tutoría en la educación superior. Arbizu Bakaikoa, Lobato Fraile y del Castillo (2005) definieron la tutoría como "una acción de intervención formativa destinada al seguimiento de los estudiantes $y$ que es considerada una actividad docente más" (p.8).

Por su parte, De la Cruz Flores, García Campos y Abreu Hernández (2006) la describen como el "proceso formativo de carácter sociocognoscitivo, personalizado y dirigido a convertir a los novatos en individuos competentes, mediante su integración a comunidades de práctica y redes de expertos, que resuelven problemas en ambientes dinámicos y complejos, crean y recrean la acción profesional y, en su caso, generan conocimiento avanzado" (p. 1367).

Herrera Aponte (2006) la expone como un "proceso dialéctico, de interacción, intersubjetivo, en donde convergen sentimientos y conocimientos en un juego de emociones y lenguaje $[\ldots]$
La tutoría como proceso de acompañamiento es tarea de compromisos y responsabilidades compartidas [...] Para lograr el éxito hay que conjugar la confianza, la comunicación, la comprensión y el respeto de ambas partes"(p.200).

En el 2008, el Centro de Investigación y Documentación Educativa (España) recuperó diversas definiciones del concepto, concluyendo que a partir de ellas, "se puede señalar la importancia de la tutoría como parte complementaria de la labor docente, ya que tiene como finalidad principal facilitar los procesos de enseñanza-aprendizaje de los alumnos y contribuir a su desarrollo personal, profesional y social" (p. 211).

\section{Tutor}

Para Arbizu Bakaikoa, Lobato Fraile y del Castillo (2005), el rol del tutor dependerá del modelo de tutoría adoptado (estos autores distinguen tres modelos, ya descritos arriba). En el modelo de tutoría integral el rol del tutor consiste en las siguientes funciones:

- "Analizar, junto con el propio alumno, sus competencias y rendimiento académico del alumno con el objeto de orientarle adecuadamente hacia un proyecto de formación.

- Facilitar información académica al alumno y hacer un seguimiento y supervisión de sus procesos de aprendizaje.

- Orientar al alumno en la construcción y gestión de su propio aprendizaje.

- Desarrollar tareas de información y orientación sobre aspectos académicos, profesionales y del mundo laboral.

- Orientar al alumno en su itinerario profesional.

- Estimular la formación continua y ocupacional del alumno.

- Atender y escuchar a los alumnos que tienen alguna problemática personal que afecte a su rendimiento académico. 
- Dirigir y asesorar al alumno hacia los servicios especializados existentes (servicio de orientación, servicios psicopedagógicos, bolsas de trabajo...).

- Atender de modo personal al alumno que solicita ayuda.

- Potenciar en el alumno su propio conocimiento, su autoestima, el desarrollo de habilidades sociales...

• Educar al alumno en valores" (p. 11).

\section{Tutorado}

Ya sea en una atención individualizada o como parte de un grupo, se debe reconocer que la función de las instituciones escolares está dirigida a ayudar a los estudiantes en su desarrollo y por tanto, ellos son el destinatario esencial de la labor tutorial.

El enfoque centrado en el aprendizaje, permite clarificar con mayor precisión la necesidad de orientar los esfuerzos a la consecución del perfil de egreso que propone la RIEMS y el plan de estudios del Bachillerato General Estatal.

De parte de los estudiantes, conforme a la naturaleza y características que tenga cada institución, se espera que asuman:

a) Comprometerse con su tutor en el desarrollo de las actividades que acuerden conjuntamente y ser consciente de que el único responsable de su proceso de formación es el propio alumno.

b) Participar en los procesos de evaluación del trabajo tutorial, de acuerdo con los mecanismos institucionales establecidos.

c) Participar en las actividades complementarias que se promuevan dentro del programa tutorial.

\section{Tipos de tutoría}

Por la finalidad que se persigue, que es el desarrollo integral de los estudiantes, la tutoría en los planteles del Bachillerato General Estatal debe ser del primer tipo: a) Preventiva. Cuando se trabaja sobre aspectos que, a la larga, puedan influir en el proceso a aprendizaje y por tanto de aprovechamiento del estudiante. El plan de acción tutorial de las escuelas (PAT) debe incluir actividades preestablecidas que sirvan de manera general para esta función. Este tipo de tutoría debe llevar una fase Diagnóstica, en la que se deberán detectar aquellas necesidades y deficiencias individuales o grupales en las que es necesario intervenir. Este es el tipo de tutoría ideal, ya que pretende detectar necesidades y carencias de los estudiantes antes de pasar a aspectos de tipo remedial. Este tipo de tutoría es para todos los estudiantes del plantel educativo. Cuando ya se ha detectado tarde una necesidad o hecho específico, se propone aplicar la tutoría remedial, misma que es tratada de forma individual y se denomina así:

b) De seguimiento. Esta pretende observar la situación problemática, su evolución y aplicar acciones que permitan, en lo posible, darle solución.

\section{Tipos de atención}

De acuerdo a la caracterización y a los actores de la acción tutorial, en el contexto del Bachillerato General se consideran dos tipos de atención, mismos que se describen a continuación:

Grupal Es aquella en la que la persona responsable de la Tutoría proporciona acompañamiento a la totalidad de alumnos de un grupo durante su permanencia en el plantel, teniendo como base un propósito formativo.

En este tipo de tutoría se trabajan aspectos cuyo tratamiento es viable a nivel grupal, tales como: trabajo cooperativo, técnicas de estudio, habilidades cognitivas, estilos de aprendizaje, por señalar algunos; a través de actividades grupales, pláticas o conferencias, test, técnicas de grupo, etc.

Individual Es aquella que brinda acompañamiento a un sólo alumno o alumna durante el proceso formativo, debido a que se requiere de un tratamiento personalizado. En ésta, se realiza una entrevista personal periódica, ya que se le da continuidad al caso, y si es necesario, al estudiante se le canaliza al ámbito institucional que le brinde el servicio profesional requerido. 


\section{Temáticas que aborda la tutoría}

La DGB marca como principales temas para abordar desde la tutoría los siguientes (DGB,2012):

- Diagnóstico.

- Autodiagnóstico.

- Metacognición.

- Estilos de Aprendizaje.

- Habitos de estudio.

- Habilidades.

- Actitudes.

- Inventario de Intereses.

- Aptitudes.

- Motivación.

- Habilidades Cognitivas.

- Atención.

- Memoria.

- Comprensión lectora.

- Administración del tiempo.

- Técnicas de Estudio.

\section{Competencias genéricas que trabaja la tutoría}

Se conoce y valora a sí mismo y aborda problemas y retos teniendo en cuenta los objetivos que persigue. Es sensible al arte y participa en la apreciación e interpretación de sus expresiones en distintos géneros. Elige y practica estilos de vida saludables. Escucha, interpreta $\mathrm{y}$ emite mensajes pertinentes en distintos contextos mediante la utilización de medios, códigos y herramientas apropiados. Desarrolla innovaciones y propone soluciones a problemas a partir de métodos establecidos. Sustenta una postura sobre temas de interés y relevancia general, considerando otros puntos de vista.
Aprende por iniciativa e interés propio a lo largo de la vida.

Participa y colabora de manera efectiva en equipos diversos.

Participa con una conciencia cívica y ética en la vida de su comunidad, región, México y el mundo.

Mantiene una actitud respetuosa hacia la interculturalidad y la diversidad de creencias, valores, ideas y prácticas sociales.

Contribuye al desarrollo sustentable de manera crítica, con acciones responsables (DGB,2012)

\section{Metodología}

\section{Tipo de investigación}

Estudio cuantitativo de tipo descriptivo, pues busca describir una situación y se realiza a través de encuestas para medir algún factor específico.

\section{Muestra}

Se encuestó a 105 asistentes a la Tercera Expo Tutoría, participantes de ambos sexos tutores, docentes y asistentes.

\section{Instrumento}

Fue una encuesta diseñada con el objetivo de conocer la opinión del evento y se preguntó sobre el tema de interés para abordarse en los siguientes eventos.

\section{Resultados}

Los resultados muestran que los temas de mayor interés son violencia (famliar, escolar y en el noviazgo) $19 \%$ y primeros auxilios médicos con el mismo porcentaje (19\%).

Seguido de éstos se mencionan los temas de sexualidad y estrés con $16 \%$ respectivamente, seguido de orientación vocacional y drogas con un $9 \%$ cada uno. Por último, mencionan la relación maestro alumno y autoestima con un $6 \%$ respectivamente (ver Gráfico 1) 


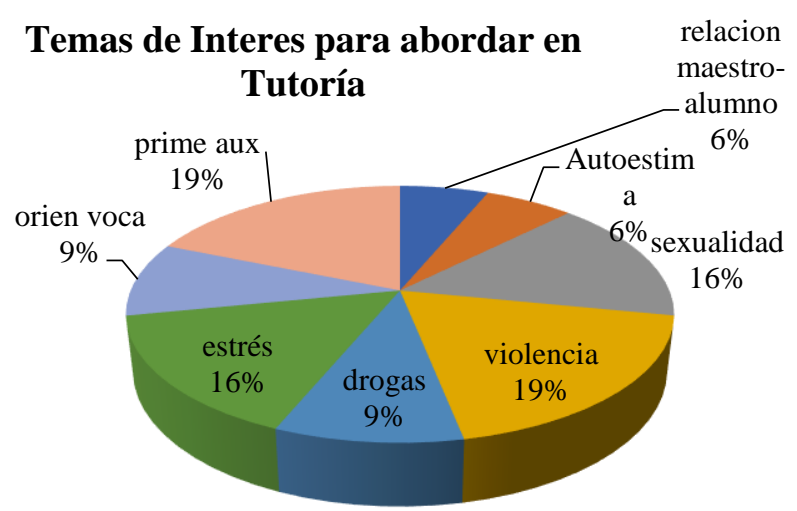

Gráfico 1

Fuente: elaboración propia

\section{Conclusiones}

Los temas centrales de la Tutoría tanto grupal como individual, son los relacionados con el desempeño académico y herramientas que les permitan desempeñarse de manera más apropiada y eficiente en sus diversas asignaturas, si bien, los resultados de este trabajo muestran que, además de lo educativo, en la actualidad los tutores muestran desafíos al manejar el área personal y social del estudiante. Esto se deduce con los temas que proponen para futuras capacitaciones que en lo general, se enfocan en el área personal del joven, debido a que problemas como noviazgo, drogas, sexualidad, etc., son temas que afectan el nivel de aprovechamiento escolar, y por ello, se les debe capacitar para orientar en medida de lo posible a los jóvenes, sin dejar de lado, el apoyo y canalización con el departamento psicopedagógico, pero el apoyar como primer instancia es de vital importancia debido a la interacción y confianza tutor-alumno.

\section{Referencias}

Arbizu, F., Lobato, C., y Del Castillo, 1. (2005). Algunos modelos de abordaje de la tutoría universitaria. Revista de Psicodidáctica, 10(1), 7- 21.2 Recuperado de http://dialnet.unirioja.es/servlet /articulo?codigo $=1222702$

Centro de Investigación y Documentación Educativa. (2008). La orientación en la atención a la diversidad; los procesos de enseñanzaaprendizaje y la acción tutorial. En M. Grañeras
De la Cruz, G., García, T. y Abreu, L. (2006). Modelo integrador de la tutoría: de la dirección de tesis a la sociedad del conocimiento. Revista Mexicana de Investigación Educativa, 11(31), 1363-1388. Recuperado de http://dialnet.unirioja.es/servlet/articulo?codigo $=2323338$ DE LA CRUZ FLORES, G.,CHEHAYBARY KURY

Herrera Aponte, M. (2006). El proceso de tutoría. Un ritual a la esperanza. EDUCARE, 10(3), 192-201. Recuperado de http://revistas.upel.edu.ve/index. php/educare/article/view/145

Pastrana \& A. Parras Laguna (Coords.), Orientación educativa: fundamentos teóricos, modelos institucionales y nuevas perspectivas (pp. 181-224). Madrid: Ministerio de Educación, Política Social y Deporte, Subdirección General de Información y Publicaciones.

Romo, A. (2011) La tutoría: una estrategia innovadora en el marco de los programas de atención a estudiantes México, D.F.: Asociación Nacional de Universidades e Instituciones de Educación Superior, Dirección de Medios Editoriales, 2011. 123 p. - (Colección Cuadernos Casa ANUIES) 